\title{
2 \\ Four Facts About ALL Statistics
}

There are four very important facts about nonparametric statistics, and all statistics in general, that you should know:

1. Statistics in this book have ABSOLUTELY nothing to do with advanced mathematics. It is unfortunate that statistics has been lumped with advanced mathematics. Some (very) basic knowledge of math is required, but that's all. Until the mid-1970s, complex looking formulas filled the pages of statistics books. All of those formulas were nothing more than computational short cuts, conveniences for people who had to compute values with pencil and paper. Advanced math provided those conveniences. ANY shorter and/or easier formulae were welcome. The computer changed that, freeing people from tedious, error-prone calculations. Statistics books began to change, focusing more on the use of computers to calculate statistics to support the decision-making process, and less on formulae and short-cuts.

2. Statistics NEVER MADE A DECISION! All statistics (of any kind) can ever do is lend support and analysis to the decision that you, the decision-maker, have to make. Make good decisions: you get promoted. Make bad decisions: you get fired. And if anyone asks, you can support your decision by showing the analysis you have done.

3. It is INCUMBENT UPON YOU, the decision maker, to be certain that the correct statistical analysis procedure is used. There never has been, nor will there ever be, a procedure to select the "correct" statistical procedure. The toolbox analogy works well here: Statistics is a toolbox full of tools. It is up to you to select the correct tool and to use it correctly.

4. Because ALL statistics are estimates, they are not EXACTLY what you wish to know. But that fact should not bother you because statistics can still yield good information. Statistics are ESTIMATES of what is really going on in the population. And if you follow what is presented here, the estimates you generate will be very close to the unknown population value that you wish you could know. 